\title{
NEUTRINO MASSES, NEUTRINO OSCILLATIONS AND DARK MATTER *
}

\author{
HAIM HARARI \\ Weizmann Institute of Science \\ 76100 Rehovot \\ Israel.
}

\begin{abstract}
We discuss bounds on neutrino masses using an analysis based on direct measurements, cosmological bounds, oscillation experiments, the solar neutrino puzzle and theoretical considerations on neutrino decays. We present four possible solutions for the mass range of the three neutrino flavors. We outline experiments which can distinguish among these solutions and discuss their implications for the cosmological dark matter problem.
\end{abstract}

\section{Introduction}

In this report we discuss several issues related to neutrino masses, neutrino oscillations, neutrino decays and neutrinos as dark matter candidates. None of these have ever been convincingly observed. But neutrino physics is largely an art of learning a great deal by observing nothing. It can give us extremely useful information on physics beyond the standard model and can help us probe, indirectly, higher energy scales and cosmological problems.

Much of the literature on neutrino masses, oscillations and decays is based on very specific theoretical models (e.g. GUTS, left-right symmetry, string inspired models, majoron schemes, etc.). Within the framework of a well defined model it is sometimes possible to make specific predictions concerning various physical quantities. However, in the absence of direct observations of masses, mixing or decays, such predictions are often less than useful. At the other extreme we find general "theorems" or arguments based on "the most general case". These are often useless and lead to very weak predictions.

Our approach here will be different. We start by defining a simple reasonable theoretical framework, motivated by plausibility arguments. It will be much more general than any specific detailed model, but not general enough to become useless. We than review all the information that we can collect on the interrelated problems of neutrino masses, oscillations and decays. We use information

* Supported in part by the Israel Commission for Basic Research, the U.S.-Israel Binational Science Foundation, and the Minerva Foundation 
from direct mass measurements, cosmology, oscillation experiments, theoretical arguments, comparison with non-neutrino experiments and solar neutrino rates. When we combine all of this information and use our plausible theoretical framework, we reach surprisingly strong conclusions concerning neutrino properties. We are also led to related interesting speculations concerning the cosmological dark matter problem and we discuss crucial experiments which can answer some of the open questions. Of particular interest to us is a $\nu_{\mu}-\nu_{\tau}$ oscillation experiment which may prove or disprove the hypothesis that $\nu_{\tau}$ is responsible for the cosmological dark matter.

Much of the work discussed here is based on collaboration ${ }^{[1]}$ with Y. Nir, to whom I am indebted for many helpful discussions. An earlier version of this report was included in the Proceedings of the Neutrino 88 conference ${ }^{[2]}$, and the last section is based on a recent proposal ${ }^{[3]}$ for a $\nu_{\mu}-\nu_{\tau}$ oscillation experiments.

\section{The See-Saw Mechanism for Neutrino Masses and a Speculation on Neutrino Mass Ratios}

Neutrinos are either exactly massless or extremely light. They are lighter than the corresponding charged leptons at least by several orders of magnitude and, as we will show below, probably by seven or more orders of magnitude.

There is no convincing explanation for exactly massless neutrinos. In the minimal standard model one simply declares that there is no right handed neutrino. In that case, the neutrino mass is precluded by chiral symmetry. However, this really amounts to assuming the answer, rather than predicting it. Much worse - even if we accept an exactly massless neutrino in the standard model, practically any physics beyond the standard model, will reintroduce the neutrino mass. The only known exception is minimal SU(5) which both disagrees with experiment and leaves crucial issues (hierarchy problems, generation puzzle) totally unexplained.

An exactly massless neutrino requires an absolute new symmetry which remains unbroken to all orders both within the standard model and in any new physics beyond it. No one has suggested such a symmetry. There is no good reason to believe that neutrinos are indeed exactly massless.

If neutrinos are approximately massless, i.e. they have a negligible mass with respect to other leptons and quarks, we must again insist on a simple explanation. It should tell us how certain fermions can be much lighter than others. Whatever that explanation is, it should single out the neutrinos and clarify why it is them, and no other fermions, which are so light. In that respect, the suggestion that neutrinos have Dirac masses and that these masses just "happen to be small" (with a Yukawa coupling smaller than $10^{-10}$ for $\nu_{e} !$ ), is totally unacceptable.

Fortunately, there is one simple and elegant theoretical framework ${ }^{[4]}$ which explains why neutrinos, and only neutrinos, are much lighter than all other fermions. This framework was first proposed in the context of GUTs, but it 
has no necessary relation to GUTs. It is much more general, and may appear naturally in practically any theory beyond the standard model.

The idea is simple: neutrinos, like any other fermion, have Dirac masses which are assumed to be of the usual order of magnitude. Neutrinos, unlike any other fermion in the standard model, may also have a Majorana mass. The Majorana mass of a left-handed neutrino may only come from a Higgs triplet (because $\nu_{L}$ is an $I_{3}=\frac{1}{2}$ member of a weak isodoublet and a $\nu_{L} \nu_{L}$ term must therefore transform like an $I_{3}=1$ component of a triplet, coupling only to Higgs isotriplet). Higgs triplets are unwanted, unnecessary and, if they existed, they would have spoiled the mass relation $M_{W}=M_{Z} \cos \theta_{W}$. In contrast, the Majorana mass of a right-handed neutrino may come only from a Higgs singlet (because $\nu_{R}$ is an $I=0$ singlet, hence $\nu_{R} \nu_{R}$ can only couple to a Higgs isosinglet). Higgs singlets are harmless to the standard model. They do not contribute to the $\mathrm{W}$ or $\mathrm{Z}$ mass and do not influence $M_{W}=M_{Z} \cos \theta_{W}$. But Higgs singlets are actually present and are practically necessary in any theory beyond the standard model. If such a theory is based on any symmetry which is larger than $\mathrm{SU}(3) \times \mathrm{SU}(2) \times \mathrm{U}(1)$ (e.g. GUTs, left-right symmetry, technicolor, horizontal symmetry, most composite models), the higher symmetry will be broken down to the standard model symmetry by a Higgs field which is a non-singlet under the higher symmetry (and therefore breaks it) but is a singlet under the standard model gauge group (and therefore preserves it). Such a singlet Higgs will have a vacuum expectation value of order $\Lambda$, where $\Lambda$ is some new energy scale of the new "beyond standard" physics. We know that $\Lambda$ can be anywhere between, say, $\mathrm{O}(\mathrm{TeV})$ and $\mathrm{M}_{\text {planck. Neutrinos, }}$. unlike charged leptons and unlike quarks, could then have a "normal-size" Dirac mass and a large right-handed Majorana mass. This situation is logical, natural and almost inescapable in many models beyond the standard model. It is a situation which is unique for neutrinos and does not apply to other leptons or quarks. It leads to a simple explanation for the small mass of the observed left-handed neutrinos.

The above scenario leads to $2 \times 2$ mass matrix ${ }^{[4]}$ for each generation of neutrinos. Ignoring generation mixing (we will return to it in a minute) we obtain:

$$
\left(\begin{array}{cc}
0 & m_{D} \\
m_{D} & M
\end{array}\right)
$$

where $m_{D}$ is a Dirac mass assumed to be comparable to the mass of the corresponding charged lepton and $\mathrm{M}$ is a Majorana mass due to a Higgs singlet and driven by a new energy scale $\Lambda$. This is the famous "see-saw" matrix.

In the quark case, quarks in the same generation have mass ratios $\frac{m_{u}}{m_{d}} \sim 0.55 ; \quad \frac{m_{c}}{m_{s}} \sim 9 ; \quad 15 \leqslant \frac{m_{t}}{m_{b}} \leqslant 40$. We therefore guess that $m_{D}$ is roughly within one order of magnitude of $m_{\ell}$, the mass of the corresponding charged lepton. 
The two eigenvalues of the mass matrix are:

$$
m_{1} \sim \frac{m_{D}^{2}}{M} ; m_{2} \sim M
$$

The first eigenstate (with mass $m_{1}$ ) is almost purely left-handed with a small right-handed admixture, determined by the small ratio $\frac{m_{D}}{M}$. Similarly, the heavy eigenstate is almost purely right-handed. The ratio $\frac{m_{D}}{M}$ is at most $10^{-3}$ (for $\frac{m_{\tau}}{1 T e V}$ ) but could be much smaller (e.g. $\frac{m_{e}}{M_{G U T}} \sim 10^{-18}$ ).

Assuming $m_{D} \sim O\left(m_{\ell}\right)$ and $M \sim O(\Lambda)$, the light eigenstates is much lighter than an ordinary lepton:

$$
m_{1} \sim \frac{m_{D}^{2}}{M} \sim \frac{m_{\ell}^{2}}{\Lambda} \sim m_{\ell}\left(\frac{m_{\ell}}{\Lambda}\right) \ll m_{\ell}
$$

That is the "see-saw" explanation ${ }^{[4]}$ for the lightness of the neutrino. The heavy eigenstate is much heavier than an ordinary lepton and its mass could be anywhere between $\mathrm{O}(\mathrm{TeV})$ and the Planck scale.

The approximate relation $m_{\nu} \sim \frac{m_{\Lambda}^{2}}{\Lambda}$ tells us that for a higher energy scale of the new physics, we obtain smaller neutrino masses. For instance, for $m_{\ell} \sim m_{e} \sim O(\mathrm{MeV})$ we obtain $m\left(\nu_{e}\right) \sim O(\mathrm{eV})$ for $\Lambda \sim O(\mathrm{TeV})$; but $m\left(\nu_{e}\right) \sim 10^{-12} \mathrm{eV}$ for $\Lambda \sim M_{G U T}$. It is interesting that $\Lambda \sim O(\mathrm{TeV})$ is the next achievable frontier of high energy physics and, at the same time, $m\left(\nu_{e}\right) \sim \mathrm{O}(\mathrm{eV})$ is one order of magnitude away from the direct mass measurements, neutrinoless double beta decay and the limit derived from SN1987A. A peculiar coincidence!

If the $\Lambda$-scale is identical for all three generations and if all generation mixing angles are small, the light neutrino masses are proportional to the squared masses of the corresponding leptons. Thus, regardless of the value of $\Lambda$, but as long as $\Lambda$ is generation independent, we obtain:

$$
m\left(\nu_{e}\right): m\left(\nu_{\mu}\right): m\left(\nu_{\tau}\right) \sim m_{e}^{2}: m_{\mu}^{2}: m_{\tau}^{2}
$$

This result could be modified by many factors. The relation $M \sim O(\Lambda)$ may be corrected by unknown Yukawa couplings. The relation $m_{\ell} \sim m_{D}(\nu)$ could easily be wrong by an order of magnitude.

The $\Lambda$ values or the Yukawa couplings which determine the Majorana masses of different generations can be quite different from each other. For instance: we may have $\Lambda_{e}: \Lambda_{\mu}: \Lambda_{\tau} \sim m_{e}: m_{\mu}: m_{\tau}$, in which case:

$$
m\left(\nu_{e}\right): m\left(\nu_{\mu}\right): m\left(\nu_{\tau}\right) \sim m_{e}: m_{\mu}: m_{\tau}
$$

The nondiagonal elements of the $3 \times 3$ Dirac mass matrix and/or the $3 \times 3$ Majorana mass matrix may also change the situation. We therefore consider the above pattern as very qualitative. We actually need to assume only: 
(i) The see-saw mechanism operates.

(ii) The light neutrino mass is within a few orders of magnitude from $\frac{m_{D}^{2}}{\Lambda}$.

(iii) The neutrino masses obey ratios:

$$
m\left(\nu_{e}\right): m\left(\nu_{\mu}\right): m\left(\nu_{\tau}\right) \sim m_{e}^{P}: m_{\mu}^{P}: m_{\tau}^{P}
$$

where the power $\mathrm{P}$ obeys $1 \leqslant P \leqslant 2$ (we may even relax this inequality to $\frac{1}{2} \leqslant P \leqslant 3$, without changing too many conclusions).

These are very weak and reasonable assumptions. We will refer to them as the "reasonable see-saw" scenario ${ }^{[1]}$.

Note that we have not assumed anything about the explicit theoretical model leading to the "see-saw". Neither GUTS nor right-handed currents are absolutely necessary.

Our "reasonable see-saw" assumption leads, among other things, to the rough estimate

$$
O(10) \varsigma \frac{m\left(\nu_{r}\right)}{m\left(\nu_{\mu}\right)} \lesssim O\left(10^{3}\right)
$$

We will see that this relation, if true, leads to far-reaching consequences.

\section{Experimental Bounds on Neutrino Masses and Oscillations} masses $^{[5]}$ :

Direct measurements have yielded the following bounds on neutrino

$$
m\left(\nu_{e}\right)<18 \mathrm{eV} ; m\left(\nu_{\mu}\right)<250 \mathrm{keV} ; m\left(\nu_{\tau}\right)<35 \mathrm{MeV}
$$

To these we may add the bound derived from SN1987A, an almost direct bound. Depending on various assumptions, values between 10 and $20 \mathrm{eV}$ are obtained as the upper bound on $m\left(\nu_{e}\right)$ from SN1987A.

A fourth generation of neutrino may exist, although the well-known argument on nucleosynthesis indicates that a fifth light neutrino is ruled out and a fourth one is not very likely ${ }^{[6]}$.

It is interesting that the present direct experimental bounds obey ratios which are not too different from those of the squared masses of the corresponding charged leptons. For instance, if $m\left(\nu_{e}\right): m\left(\nu_{\mu}\right): m\left(\nu_{\tau}\right)=m_{e}^{2}: m_{\mu}^{2}: m_{\tau}^{2}$ and if $m\left(\nu_{\tau}\right)$ were found to be exactly at its present experimental bound of $35 \mathrm{MeV}$, we would expect $m\left(\nu_{\mu}\right) \sim 120 \mathrm{KeV}, m\left(\nu_{e}\right) \sim 3 \mathrm{eV}$. Hence, the unlikely possibility that all three neutrino masses are not too far from their present direct mass limits $^{[7]}$ is compatible with the reasonable see-saw, provided that $M$ is actually around $100 \mathrm{GeV}$ or so. Of course, there is no good reason to expect nature to choose such a possibility.

Most of the information concerning leptonic mixing angles is obtained from neutrino oscillations experiments. There have been several unconfirmed claims of two-standard-deviation effects in various oscillation experiments, but all of them were contradicted by later experiments. A given negative oscillation experiment 
can only rule out a region in the $\Delta_{i j}-\sin ^{2} 2 \theta_{i j}$ plane, where $\Delta_{i j}=m_{i}^{2}-m_{j}^{2}, m_{i}$ and $m_{j}$ are the masses of the mixed neutrinos and $\theta_{i j}$ is their leptonic mixing angle. Consequently, no value of $\theta_{i j}$ can be completely excluded. It can be excluded only for a specific range of $\Delta_{i j}$-values.

We have no theoretical information concerning the values of the leptonic mixing angles. The three quark mixing angles are

$$
\theta_{12}=0.22, \theta_{23}=0.043 \pm 0.008, \theta_{13} \leq 0.01
$$

or:

$$
\sin ^{2} 2 \theta_{12}=0.18, \sin ^{2} 2 \theta_{23} \sim 0.005-0.01, \sin ^{2} 2 \theta_{13} \leq 4 \times 10^{-4} .
$$

One possible guess is that the leptonic mixing angles are roughly comparable to the quark angles. It is possible to support this guess by the following hand waving argument: It is very likely that there are relations between fermion masses and mixing angles. Many models actually indicate that a given mixing angle between two generations is somehow related to the mass ratio of the fermions in the same two generations. The detailed formulae vary from one model to another. The angles may be proportional to mass ratios or to square roots of mass ratios, etc. However, given the mass ratios, the angles are determined in all such models.

The mass pattern of the three charged leptons is not very different from that of the quarks. If fermion mass ratios indeed determine the mixing angles, one might therefore expect that the leptonic mixing angles are within, say, an order of magnitude of the quark angles.

If we explicitly assume that $\theta_{\boldsymbol{e} \mu}=\theta_{12}^{q} \sim 0.22$, we conclude from oscillation experiments that $\Delta_{12} \leqslant 0.1 \mathrm{eV}^{2}$. If we assume $\theta_{\mu \tau}=\theta_{23}^{q} \sim 0.043$, we conclude from experiment that $\Delta_{23} \lesssim 1 \mathrm{eV}^{2}$. However, if $\theta_{\mu \tau}$ is slightly smaller (say 0.03 ) we have no bound at all on $\Delta_{23}$. We will return to this subject towards the end of our discussion.

The well-known MSW effect ${ }^{[8]}$ may explain the deficiency of solar neutrinos in the Davis experiment ${ }^{[9]}$. The Bethe solution requires ${ }^{[10]}$

$\Delta_{i j} \sim 10^{-4} \mathrm{eV}^{2}$, i.e. $m\left(\nu_{\mu}\right)$ or $m\left(\nu_{\tau}\right)$ of order $10^{-2} \mathrm{eV}$. The Rosen-Gelb solution ${ }^{[11]}$ allows $\Delta_{i j}$-values ranging between $10^{-7}-10^{-4} \mathrm{eV}^{2}$, depending on $\theta_{i j}$. Consequently, in all solutions, the heavier neutrino mass $\left(\nu_{\mu}\right.$ or $\left.\nu_{\tau}\right)$ is between $10^{-4} \mathrm{eV}$ and $10^{-2} \mathrm{eV}$. All of these values are well below the bounds obtained from direct neutrino oscillation experiments in accelerators or reactors. If $m\left(\nu_{\mu}\right) \sim O\left(10^{-2}\right.$ $\mathrm{eV})$, the see-saw mechanism yields $\Lambda \sim O\left(10^{9} \mathrm{GeV}\right)$. For $m\left(\nu_{\tau}\right) \sim O\left(10^{-2} \mathrm{eV}\right)$, we obtain $\Lambda \sim O\left(10^{12} \mathrm{GeV}\right)$. 


\section{Cosmological Bounds}

A well known cosmological argument tells us that ${ }^{12)}$

$$
\sum_{i} N_{\mathrm{o}}\left(\nu_{i}\right) m\left(\nu_{i}\right)<\rho_{0}=\Omega \rho_{c}=\Omega \cdot \frac{3 H_{\mathrm{o}}^{2}}{8 \pi G}=\Omega h^{2} \cdot 11 \frac{\mathrm{KeV}}{\mathrm{cm}^{3}}
$$

where $\nu_{i}$ is a neutrino flavor, $N_{\mathrm{o}}$ is the number density of $\nu_{i}, \rho_{\mathrm{o}}$ is the present density of the universe, $\rho_{c}$ is the critical density corresponding to a flat universe, $H_{\mathrm{o}}$ is the Hubble parameter, $G$ is Newton's constant and $h=H_{\circ} / 100 \mathrm{~km} / \mathrm{sec} / \mathrm{Mpc}$.

For the accepted values of $\Omega \leq 2 ; 0.5 \leq h \leq 1 ; 10^{10} \leq t_{\mathrm{o}} \leq 2 \cdot 10^{10}$ yrs ( $t_{\circ}=$ present age of the universe) we obtain $\Omega h^{2} \leq 0.65$.

The neutrino decoupling temperature is $\mathrm{O}(\mathrm{MeV})$. For $m(\nu)<O(\mathrm{MeV})$, all neutrino species have a number density $\frac{3}{11} N_{\mathrm{o}}(\gamma)$ where $N_{\mathrm{o}}(\gamma) \sim 400 \mathrm{~cm}^{-1}$ is the number density of photons. We then obtain:

$$
m\left(\nu_{i}\right)<100 \Omega h^{2} \leq 65 \mathrm{eV}
$$

For $m(\nu)>O(\mathrm{MeV})$, each number density is suppressed by an appropriate Boltzman factor $e^{\frac{-m\left(\nu_{i}\right)}{k T}}$. A more complicated treatment is needed, leading to a lower limit ${ }^{13)}$ (for $\Omega h^{2} \leq 0.65$ ):

$$
m\left(\nu_{i}\right) \geq 4.2 \mathrm{GeV}
$$

All mass values between $65 \mathrm{eV}$ and $4.2 \mathrm{GeV}$ are thus excluded, under very general assumptions which essentially depend only on the gross features of Big Bang Cosmology. The only strong assumption hidden in the argument is the assumption that neutrinos are stable. We return to this issue below. However, if we ignore the possibility of neutrino decays for a brief moment, we must conclude that $\nu_{\mu}$ and $\nu_{\tau}$ are lighter than $65 \mathrm{eV}$. If we add the "reasonable see-saw" assumption we find:

$$
m\left(\nu_{\tau}\right)<65 \mathrm{eV} ; m\left(\nu_{\mu}\right)<f e w \mathrm{eV} ; m\left(\nu_{e}\right)<10^{-2} \mathrm{eV}
$$

This is an extremely strong result. It means that $\nu_{r}, \nu_{\mu}$ and $\nu_{e}$ are lighter than their present direct mass limits by approximately six, five and three orders of magnitude, respectively. If it holds, it means that all direct experiments of neutrino mass measurements are absolutely hopeless for the foreseeable future. This far-reaching conclusion can be avoided only if neutrinos decay. We now turn to this possibility.

The cosmological bounds $m\left(\nu_{i}\right)<65 \mathrm{eV}$ or $m\left(\nu_{i}\right)>4.2 \mathrm{GeV}$ hold only for stable neutrinos. They are based on the assumption that the energy density due to neutrinos today is related to the density at the decoupling temperature by the simple proportionality

$$
\rho \propto \frac{1}{R^{3}}
$$


i.e. as the universe expands the density is inversely proportional to the volume.

If neutrinos are unstable, the density behaves differently. After the decay, the universe becomes "radiation dominated", obeying:

$$
\rho \propto \frac{1}{R^{4}}
$$

Consequently, the density at the decay time may be larger than the density allowed for stable neutrinos. The unstable neutrinos may therefore have a higher mass. For shorter neutrino lifetime, the effect becomes larger.

A straightforward analysis ${ }^{[14]}$ yields the following bounds on the masses of unstable neutrinos ${ }^{[1]}$ :

$$
\begin{aligned}
& m_{\nu}^{2} \tau_{\nu} \leq 2 \times 10^{20} \mathrm{eV}^{2} \cdot \sec \text { for } m_{\nu}<O(\mathrm{MeV}) \\
& m_{\nu}^{-4} \tau_{\nu} \leq 1.5 \times 10^{-22} \mathrm{eV}^{-4} \text { for } m_{\nu}>O(\mathrm{MeV})
\end{aligned}
$$

These bounds mean that a neutrino may be heavier than $65 \mathrm{eV}$ provided that its lifetime is short enough. In particular, $m\left(\nu_{\tau}\right)$ may be close to $35 \mathrm{MeV}$ if $\tau\left(\nu_{\tau}\right) \leq 8 \mathrm{yrs}$ and $m\left(\nu_{\mu}\right)$ may be around $250 \mathrm{keV}$ if $\tau\left(\nu_{\mu}\right) \leq 100 \mathrm{yrs}$.

There is no reason to expect neutrinos to be stable. In fact, if the neutrinos have masses, it is almost certain that $\nu_{\tau}$ and $\nu_{\mu}$ decay into lighter neutrinos. If that is the case, it would appear that the $65 \mathrm{eV}$ cosmological limits is not valid and our strong conclusion of the previous section is unapplicable.

However, before we jump into such a conclusion, we must consider the possible rates for different neutrino decay modes. Only if these rates are sufficiently high, the $65 \mathrm{eV}$ bound is evaded. If neutrinos are unstable but decay slowly, the $65 \mathrm{eV}$ bound remains valid. We must therefore now turn to estimate neutrino decay rates.

\section{Neutrino Decays and their Implications for the Cosmological Bounds}

A complete analysis of all possible neutrino decays in all possible models ${ }^{[1]}$ (beyond the standard model) is beyond the scope of this report. Here we briefly summarize the main results.

There are three major classes of neutrino decay modes:

(i) Radiative decays such as $\nu_{i} \rightarrow \nu_{j}+\gamma, \nu_{i} \rightarrow \nu_{j}+\gamma+\gamma, \nu_{\tau} \rightarrow \nu_{i}+e^{+} e^{-}$ where $i, j$ are neutrino flavors.

(ii) Decays into neutrinos such as $\nu_{i} \rightarrow \nu_{j}+\nu_{k}+\nu_{\ell}$ where the decay products may be neutrinos and/or antineutrinos of the same or of different flavors.

(iii) Decays into Goldstone bosons such as $\nu_{i} \rightarrow \bar{\nu}_{j}+$ majoron or $\nu_{i} \rightarrow \nu_{j}+$ familon.

Radiative neutrino decays (class (i)) have been analyzed both within the standard model ${ }^{[15]}$ and in "beyond standard" schemes. In all cases one can derive bounds on $\tau(\nu)$ which depend on the mass of the decaying neutrino. An investigation of the $m(\nu)-\tau(\nu)$ plane shows that below $m(\nu) \sim \mathrm{O}(\mathrm{MeV})$ there is no 
overlap between the region allowed by the cosmological constraints on unstable neutrinos and the region allowd by the decay rate for radiative neutrino decay. Thus, if radiative decays were the only neutrino decays we would have concluded:

$$
\begin{aligned}
& m\left(\nu_{r}\right)<65 \mathrm{eV} \text { or } \mathrm{O}(\mathrm{MeV})<m\left(\nu_{\tau}\right)<35 \mathrm{MeV} \\
& m\left(\nu_{\mu}\right)<65 \mathrm{eV} \\
& m\left(\nu_{e}\right)<65 \mathrm{eV}
\end{aligned}
$$

The next class of decays $(\nu \rightarrow 3 \nu)$ can be mediated within the standard model by $Z^{\circ}$ exchange. It can also occur in "beyond standard" theories by the exchange of a horizontal gauge boson, by the exchange of a Higgs triplet $\Delta_{L}$ (with two units of lepton number) or by some interaction among subleptons in a composite model. The $Z$ exchange contribution is too slow ${ }^{[16]}$. In all other cases we cannot calculate the decay rate directly but we can relate it to the observed experimental bound on the decay of a charged lepton into three lighter charged leptons (e.g. $\tau^{-} \rightarrow e^{-} e^{+} e^{-}$etc.). We again obtain ${ }^{[1]}$ an allowed region in the $m(\nu)-\tau(\nu)$ plane, leading to the conclusion that $m(\nu)$ is either below $65 \mathrm{eV}$ or above $\mathrm{O}(\mathrm{MeV})$.

In all of the above decay modes $m\left(\nu_{\mu}\right)$ must be lighter than $65 \mathrm{eV}$. From our "reasonable see-saw" we then conclude:

$$
m\left(\nu_{\tau}\right) \leq 10^{3} m\left(\nu_{\mu}\right) \ll M e V
$$

Hence, the possibility that $m\left(\nu_{\tau}\right)>O(M e V)$ which is allowed by the estimated $\nu_{\tau}$ decay rates and by the cosmological bound on unstable neutrinos, is incompatible with $m\left(\nu_{\mu}\right)<65 \mathrm{eV}$ and the "reasonable see-saws".

The combined result of cosmology, neutrino decays and the reasonable see-saw is, therefore:

$$
\begin{aligned}
& m\left(\nu_{\tau}\right)<65 \mathrm{eV} \\
& m\left(\nu_{\mu}\right)<f e w \mathrm{eV} \\
& m\left(\nu_{e}\right)<10^{-2} \mathrm{eV}
\end{aligned}
$$

We are back to the extremely strong result obtained earlier for the case of stable neutrinos!

There is, however, one class of decay modes which, under extremely unlikely circumstances, may allow larger neutrino masses. The decay

$$
\nu_{\mu} \rightarrow \bar{\nu}_{e}+\text { majoron }
$$

may be consistent with $m\left(\nu_{\mu}\right)$ around $50-250 \mathrm{keV}$. For that to happen ${ }^{7)}$, the Majorana mass term must be driven by a $\Lambda$-scale around $100 \mathrm{GeV}$ or less, a very unlikely proposition. In addition, the age of the universe must be at most 12 or $13 \mathrm{Gyr}$. If both condition are obeyed, one cannot exclude the weird possibility 
that all three neutrino flavors have masses near their present direct bounds, i.e.:

$$
\begin{aligned}
& m\left(\nu_{\tau}\right) \sim O(10 \mathrm{MeV}) \\
& m\left(\nu_{\mu}\right) \sim O(100 \mathrm{keV}) \\
& m\left(\nu_{e}\right) \sim O(1 \mathrm{eV}) .
\end{aligned}
$$

This small window (or "peephole") requires too many miracles to be taken seriously. However, it can be tested and excluded experimentally if the direct bound on $m\left(\nu_{\mu}\right)$ is improved by less than one order of magnitude.

We conclude: It is almost certain that:

$$
\begin{aligned}
& m\left(\nu_{\tau}\right)<65 \mathrm{eV} \\
& m\left(\nu_{\mu}\right)<f e w \mathrm{eV} \\
& m\left(\nu_{e}\right)<10^{-2} \mathrm{eV}
\end{aligned}
$$

But the remote possibility of "neutrinos at the limit" cannot be excluded.

\section{Four Interesting Possibilities For The Neutrino Mass Values, and their Implications for the Cosmological Dark Matter Problem}

Dark matter seems to exist at several different scales in the universe. ${ }^{[17]}$ Here we only discuss the cosmological dark matter, i.e. the matter responsible for an $\Omega=1$ flat universe or for $\Omega$ being not too different from one. Other forms of dark matter may or may not coincide with the cosmological dark matter. The leading particle physics candidates for the cosmological dark matter are:

(i) "Light Neutrinos". These must have a mass in the range $15-65 \mathrm{eV}$. For the most likely values of $\Omega=1$ and $t_{\mathrm{o}}=15 \mathrm{Gyr}$ we need $m(\nu) \sim 20 \mathrm{eV}$. If we take note of our combined bound from cosmology, neutrino decay estimates and the "reasonable see-saw", we must conclude that, among the known neutrinos, only $\nu_{\tau}$ can provide us with such a mass. Hence - the first dark matter candidate is $\nu_{\tau}$ with $m \sim O(20 \mathrm{eV})$.

(ii) WIMPS. These are particles with masses of order few $\mathrm{GeV}$. One such candidate is an ordinary fourth generation neutrino $\nu_{\sigma}$ with mass near $4.2 \mathrm{GeV}$. We know that a hypothetical fourth charged lepton (say, $\sigma$ ) must be lighter than $350 \mathrm{GeV}$ or else it will disturb $M_{W}=M_{Z} \cos \theta_{W}$ by too much. Hence, $\frac{m(\sigma)}{m(\tau)} \leq 200$. However, if $m\left(\nu_{\tau}\right)<65 \mathrm{eV}, \frac{m(\sigma)}{m(\tau)} \leq 200$ and $\frac{m\left(\nu_{\sigma}\right)}{m\left(\nu_{\tau}\right)} \leq\left[\frac{m(\sigma)}{m(t)}\right]^{2}$, we obtain $m\left(\nu_{\sigma}\right)<3 \mathrm{MeV}$ and the $4.2 \mathrm{GeV}$ mass is excluded. On the other hand, if $\nu_{\tau}, \nu_{\mu}$ and $\nu_{e}$ are all "at the limit", $\nu_{\sigma}$ can easily be around $4.2 \mathrm{GeV}$. Other likely WIMP's are the photino as well as a variety of other types of heavy neutrino-like objects.

(iii) Axions. Here we need a new physics scale which is around $10^{12} \mathrm{GeV}$ or perhaps a bit (but not much) lower.

As we see below, all of these possibilities may be related to our discussion of neutrino masses. 
On the basis of everything we said so far we find that there are four possible solutions corresponding to four interesting mass ranges for the neutrinos. For each of these solutions we can offer one or several crucial experimental tests. Each one of them has important implications for the cosmological dark matter problem. All our conclusions are based on our fairly general framework. We combine experimental data, simple cosmological arguments, rough estimates of neutrino decay rates and the "reasonable see-saw" assumption. Based on these assumptions we now review the four solutions:

Solution I: Neutrinos at the limit (the Glashow solution $\left.{ }^{[7]}\right)$. In this case $m\left(\nu_{\tau}\right) \sim O(10 \mathrm{MeV}) ; m\left(\nu_{\mu}\right) \sim O(100 \mathrm{keV}) ; m\left(\nu_{e}\right) \sim O(1 \mathrm{eV})$. A possible fourth neutrino $\nu_{\sigma}$ might be (if it exists) around 5-100 GeV, depending on $m(\sigma)$. A $5-\mathrm{GeV} \nu_{\sigma}$ may form the cosmological dark matter. Crucial experiments are direct mass measurements of $\nu_{\tau}, \nu_{\mu}$ and $\nu_{e}$ and neutrinoless double beta decay. All of these experiments probe the relevant mass regions. All mixing angles must be extremely small (based on present data). The new physics scale $\Lambda$ is extremely low and is uncomfortably close to $M_{W}$. Neutrino lifetimes are also "at the limit" and the dominant decay mode is into antineutrino + majoron. We consider this solution extremely unlikely and almost perverse.

Solution II: Our favorite solution is the possibility that $\nu_{\tau}$ forms the cosmological dark matter. Note that if solution I is excluded (i.e. if $m\left(\nu_{\mu}\right)<O$ $(100 \mathrm{keV}))$, the next highest allowed mass range is:

$$
\begin{aligned}
& m\left(\nu_{\tau}\right) \sim 15-65 \mathrm{eV} \\
& m\left(\nu_{\mu}\right) \sim O(1 \mathrm{eV}) \\
& m\left(\nu_{e}\right) \lesssim O\left(10^{-2} \mathrm{eV}\right) .
\end{aligned}
$$

In this case all direct mass measurements are useless. Neutrinoless beta decay is hopeless. The new scale $\Lambda$ is $\mathrm{O}(100 \mathrm{PeV})$. There is no fourth generation neutrino. The crucial experiment ${ }^{[3]}$ is a search for $\nu_{\tau}-\nu_{\mu}$ oscillations for $\Delta_{\mu \tau} \sim$ $O\left(10^{3} \mathrm{eV}^{2}\right)$ but small $\theta_{\mu \tau}$ values (below $\theta_{\mu \tau} \sim 0.05$ ) which is the present limit for the relevant $\Delta_{\mu \tau}$ value. We discuss this experiment in detail below.

Solution III: The Bethe solution ${ }^{[10]}$ for the solar neutrino puzzle. If both solution I and solution II are wrong, the next range is:

$$
\begin{aligned}
& m\left(\nu_{\tau}\right) \sim f e w e V \\
& m\left(\nu_{\mu}\right) \sim O\left(10^{-2} \mathrm{eV}\right) \\
& m\left(\nu_{e}\right)<O\left(10^{-4} \mathrm{eV}\right)
\end{aligned}
$$

The value of $\Lambda$ is around $10^{9} \mathrm{GeV}$. A possible fourth neutrino $\nu_{\sigma}$ may have a mass around $65 \mathrm{eV}$ and form (if it exists) the cosmological dark matter. Direct mass measurements, and neutrinoless double beta decay experiments are hopeless. The crucial experiments are the Gallium solar neutrino experiments and, possibly, $\nu_{\tau}-\nu_{\mu}$ oscillations. 
Solution IV: The Bethe-Rosen-Gelb solution ${ }^{[10,11]}$. If solutions I, II and III are all excluded, we reach the next interesting range in which $m\left(\nu_{\tau}\right) \sim O\left(10^{-2} \mathrm{eV}\right)$ obeying the Bethe solution ${ }^{[10]}$ for the solar neutrino puzzle. However, in that case, $m\left(\nu_{\mu}\right)$ may well be around $10^{-4}-10^{-3} \mathrm{eV}$, consistent with the Rosen-Gelb solution ${ }^{(11]}$. Either or both of these neutrinos might have resonant oscillations with $\nu_{e}$ (whose mass would be below $10^{-6} \mathrm{eV}$ ). The only doable experiments in this case are the solar neutrino experiments. No other experiment mentioned in this report has a chance. The value of $\Lambda$ is $\mathrm{O}\left(10^{12} \mathrm{GeV}\right)$, consistent with the range required by axions providing the cosmological dark matter.

It is, of course, possible that all neutrino masses are even lower than the values of solution IV. This would be very sad indeed and no known experiment can probe such a range.

Based on our reasonable and general framework we showed that there are four possible solutions for the mass range of the light neutrinos. Each of these solutions can be tested by one or more crucial doable experiments. Our prejudice (which, like all prejudices, is unfounded) it to favor solution II in which the cosmological dark matter is $\nu_{\tau}$. Solution III is probably a close second. Time and experiment will tell.

\section{A Crucial Dark Matter Experiment}

The crucial experiment which can "clinch" solution II is a very special neutrino oscillation experiments, probing $\nu_{\tau}$ masses at the level of 15-65 eV.

We are discussing here a $\nu_{\tau}$ mass value which is six orders of magnitude below the best direct limit ${ }^{[5]} m\left(\nu_{\tau}\right)<35 \mathrm{MeV}$. The only way to probe this mass region are neutrino oscillations involving $\nu_{\tau}$. Since we assumed that $m\left(\nu_{\tau}\right) \gg$ $m\left(\nu_{\mu}\right) \gg m\left(\nu_{e}\right)$, and we are interested in the range $15 \mathrm{eV} \leq m\left(\nu_{\tau}\right) \leq 65 \mathrm{eV}$, we must consider only $\nu_{\tau}-\nu_{\mu}$ and $\nu_{\tau}-\nu_{e}$ oscillations and we know that, to a good approximation, $\Delta m^{2} \approx\left[m\left(\nu_{\tau}\right)\right]^{2} \approx(200-4500) e V^{2}$.

What can we say about the $\nu_{\tau}-\nu_{e}$ and the $\nu_{\tau}-\nu_{\mu}$ mixing angles $\theta_{\tau e}$ and $\theta_{\tau \mu}$ ?

In section 3 we already speculated that quark and lepton mixing angles might be of the same order of magnitude. The angle $\theta_{\tau e}$ mixes non-adjacent generations. It is analogous to $\theta_{13}^{(q)}$ in the quark sector, which is known to be smaller (but probably not much smaller) than $10^{-2}$. If $\theta_{\tau e} \approx \theta_{13}^{(q)}$ we expect $\sin ^{2} 2 \theta_{\tau e} \leq 4 \cdot 10^{-4}$. The best $\nu_{r}-\nu_{e}$ oscillation data ${ }^{[18]}$ (as well as the best $\nu_{e}$ "disappearance" data) reach only much larger values of $\sin ^{2} 2 \theta_{\tau e}$ and therefore tell us nothing about $m\left(\nu_{\tau}\right)$.

This leaves us with $\nu_{\tau}-\nu_{\mu}$ oscillations as the last resort. The angle $\theta_{\tau \mu}$ mixes adjacent generations. It is analogous to $\theta_{23}^{(q)}$ in the quark sector. Experimentally, $\sin \theta_{23}^{(q)}=0.043 \pm 0.008$. If we had $\theta_{r \mu}=\theta_{23}^{(q)}$ we would expect $\sin ^{2} 2 \theta_{\tau \mu} \approx 0.005-0.010$. In the quark sector, we have another mixing angle 
which connects neighbouring generations: the original Cabibbo angle, obeying $\sin \theta_{12}^{(q)}=0.22$ or $\sin ^{2} 2 \theta_{12}^{(q)}=0.18$. We do not really know why $\theta_{12}^{(q)} \gg \theta_{23}^{(q)}$. We also do not know the actual value of $\theta_{\tau \mu}$, but on the basis of the above analogy to the quark sector, it might be anywhere, say, between 0.03 and 0.22 . the pattern of the charged lepton mass ratios is not very different from that of the quark mass ratios. Most theoretical models expect mixing angles to be somehow related to fermion mass ratios. We may therefore "guess" that the $\theta_{\tau \mu}$ is not far from the above range, possibly below it, but not too far below. Since $\theta_{13}^{(q)}$ is probably near 0.01 , and the mixing of "distant" generations is expected to be smaller, we propose a very conservative lower bound $\theta_{\tau \mu} \geq 0.01$. This would mean $\sin ^{2} 2 \theta_{\tau \mu} \geq 4 \cdot 10^{-4}$. This bound seems safe although, in principle, arbitrarily small values of $\theta_{\tau \mu}$ cannot be excluded. What we need is, therefore, a $\nu_{\tau}-\nu_{\mu}$ oscillation experiment probing the region of $\Delta m^{2}$ between 200 and $4500 \mathrm{eV}^{2}$ and reaching $\sin ^{2} 2 \theta_{\tau \mu}$ values which are at least as low as $4 \cdot 10^{-4}$, preferrably even lower.

The relevant range in $\Delta m^{2}$ is easily accessible. How far can we go in the other crucial variable, $\sin ^{2} 2 \theta_{\tau \mu}$ ? The best $\nu_{\mu}$ "disappearance" experiments reach only ${ }^{[8]} \sin ^{2} 2 \theta_{\mu x} \approx 0.05$, far above the required range. By far the best $\nu_{\mu}-\nu_{\tau}$ data comes from ${ }^{[9]}$ Fermilab experiment E531, using a hybrid combination of an emulsion and a spectrometer. This experiment, at the $90 \%$ confidence level, reached $\sin ^{2} 2 \theta_{\tau \mu} \approx 4 \cdot 10^{-3}$, just enough to exclude $\theta_{\tau \mu}=\theta_{23}^{(q)}$. What we now need is an improved experiment that can reach at least down to $\sin ^{2} 2 \theta_{\tau \mu} \approx 4 \cdot 10^{-4}$, hopefully below it. Such an experiment will provide us with an excellent probe of the possibility that the cosmological dark matter is due to tau-neutrinos.

The E531 experiment ${ }^{[19]}$ was not originally designed to search for $\nu_{\tau}$ oscillations. It was a by-product of a charm lifetime experiment. It still achieved, by far, the best $\nu_{\mu}-\nu_{\tau}$ oscillation data. In that experiment, approximately 4000 neutrino interactions were detected. A $\tau$ candidate was defined as an event with a kink (having $p_{T}>125 \mathrm{MeV}$ ) or a three-prong secondary vertex, no prompt muon (to eliminate standard $\nu_{\mu} \rightarrow \mu$ events), a negative charged track (to eliminate charm events) and a minimum momentum for the $\tau\left(p_{\tau}>2.5 \mathrm{GeV}\right.$, to avoid confusion with other background). With these cuts, most $\tau$ events should survive, but no candidate events were found. The experiment, with these cuts, had no background at all. On the basis of zero $\tau$ candidates and 1870 ordinary charged current events with an identified $\mu$, the range of $\sin ^{2} 2 \theta_{\tau \mu} \leq 4 \cdot 10^{-3}$ was obtained.

Improving the bound by at least an order of magnitude would require a new dedicated experiment using similar techniques. The emulsion seems necessary in order to observe $\tau$ tracks with a typical length of a few hundred microns. The spectrometer is needed in order to point towards the suspected vertex. Conceptually, the simplest method would be to repeat the essential features of experiment E531 with a larger number of events. One needs at least 20,000 charged current 
neutrino interactions with identified muons, preferrably more. Depending on the efficiency and the acceptance for muon identification, this would require a total of at least 30,000 and probably 40,000 neutrino interactions.

This can be achieved by any combination of more emulsion, higher beam intensity and longer running time. Assuming that the transverse size of the detector covers most of the width of the neutrino beam, the number of neutrino interactions can be roughly estimated by the following crude formula:

$$
\left[\frac{N_{\nu-\text { events }}}{1000}\right]=\eta \cdot\left[\frac{E_{p}}{100 \mathrm{GeV}}\right]\left[\frac{n_{p}}{10^{18}}\right]\left[\frac{M_{\text {target }}}{1 \text { ton }}\right]
$$

where $E_{p}$ and $n_{p}$ are, respectively, the energy and the number of protons on target and $M_{\text {target }}$ is the active target mass. The coefficient $\eta$ is always of order one and it contains all the details of the beam, detector, etc. In a sample of CERN and Fermilab experiments over the last few years, $\eta$-values between 0.6 and 3.5 are obtained. For our purposes, we need to generate a factor of 40 on the left hand side of our equation.

For a single realistic run at Fermilab with $800 \mathrm{GeV}$ protons and $10^{18}$ protons on target, we therefore have:

$$
\left[\frac{N_{\nu-e v e n t s}}{1000}\right]=8 \eta \cdot\left[\frac{M_{\text {target }}}{1 \text { ton }}\right] .
$$

For $\eta=1$ we therefore need, say, two runs with at least 2.5 tons of emulsion. The situation for the CERN SPS is somewhat better. Because of the higher beam intensity and the higher repetition rate of the machine, and in spite of the lower energy, one obtains for a typical realistic run $E_{p}=400 \mathrm{GeV}, n_{p}=6 \cdot 10^{18}$, yielding:

$$
\left[\frac{N_{\nu-\text { events }}}{1000}\right]=24 \eta \cdot\left[\frac{M_{\text {target }}}{1 \text { ton }}\right] .
$$

With $\eta=1$, two such runs with $800 \mathrm{~kg}$ (or 200 liters) of emulsion would do the job. Some of the above numbers could be modified by factors of two, depending on the quality of the neutrino beam, the length of the run, the percentage of machine protons dedicated to the experiment, the distance of the detector, the acceptance and efficiency, etc. In fact, we believe that by optimizing all of these parameters, it may be possible to obtain the required sensitivity with a somewhat smaller amount of emulsion, possibly below 100 liters. For $\eta \approx 3$ (a value which have been achieved in past experiments), one needs approximately 70 liters.

With so many events, scanning the emulsion becomes a difficult and lengthy procedure. Almost all scanned events would involve a muon which is detected by the spectrometer and traced back to a primary vertex in the emulsion. Rejecting these events is a fairly rapid procedure. Selecting the serious candidates and scanning them is the heart of the experiment. A dedicated $\nu_{\tau}$ experiment which is not a by-product of something else, may allow a more efficient procedure of selecting candidate events before the cuts. 
It may be worthwhile to concentrate on specific decay modes of $\tau$ (e.g. single hadron or three prongs or electron) and in this way considerably reduce the necessary amount of scanning. The price paid would, of course, be the necessity of having a higher total number of events and therefore a proportionately larger amount of emulsion.

It seems that the best method would be to concentrate on events containing an energetic negative electron and no muon. Such events would include $17 \%$ of all $\tau$-leptons, necessitating a total number of events which is six times larger, i.e. a total of 250,000 neutrino interactions. However, such a procedure would eliminate all normal charged current events and almost all neutral current events. The main physics background here would come from $\nu_{e}$ contamination in the neutrino beam, usually estimated at $1 \%$. This would yield approximately 1,500 $\nu_{e}$-initiated charged current events. Most of the scanned events would be of this type. If the electron comes from the primary vertex in the emulsion, the event should be rejected. If a kink is observed for an $e^{-}$, it is a $\tau^{-}$candidate. In spite of the sixfold increase in the total number of neutrino interactions, the absolute number of scanned events will be reduced by more than an order of magnitude, relative to the case in which one searches for all $\tau$ decay modes.

The total amount of emulsion needed for performing this version of the experiment at CERN will have to be of the order of 500 liters (assuming $\eta \approx 3$ ). The typical effective transverse area of the neutrino beam at a distance of $1 \mathrm{~km}$ is a few squared meters (say, $3 \mathrm{~m}^{2}$ ), leading to a total emulsion thickness of the order of $15 \mathrm{~cm}$ or five radiation lengths. In order to overcome showers, conversions and other facts of life, it would be advantageous to use several layers of emulsion (say, each with a depth of $1 \mathrm{~cm}$ ) separated by tracking chambers which can help identify the electrons and distinguish them from various types of background. The combined electronic information from the detector behind the emulsion and the chambers between the emulsion plates could help identify true electron events, reducing the total number of scanned events to a few thousands, a number similar to that of experiment E531. Scanning will consist of searching for the relatively simple signature of a kink involving a short track of a few hundred microns followed by a single negative electron.

It is conceivable that the experiment can also be performed with other detectors containing a track-sensitive target. It might be interesting to pursue this possibility. However, the requirement of hundreds of kilograms of active target and the necessity of observing $\tau$-tracks of a few hundred microns are not easily reconciled in other methods. A particularly attractive possibility along these lines is the idea of using scintillating optical fibres in order to detect $\tau$ tracks in a neutrino beam ${ }^{[20]}$.

It is, in principle, also possible to detect $\tau$ leptons without explicitely observing their tracks, using much larger active targets and higher event rates. However, at the level of sensitivity required here, background becomes an ex- 
tremely serious problem in such experiments.

If $\tau$ events are discovered, we must be certain that they come from $\nu_{\mu}^{\prime} s$ which oscillated into $\nu_{\tau}^{\prime} s$ rather than from a $\nu_{\tau}$-contamination which exists in the neutrino beam as a result of direct hadronic decays. The prime candidate for such decays is the $c \bar{s}$ meson, known as $F$ or $D_{s}$. The decay of $F$ is the dominant mechanism for producing $\nu_{\tau}$ in beam dump experiments. However, for the type of experiment discussed here, at a distance of, say, $1 \mathrm{~km}$, the number of $\tau$ events originating from $F$-decay is expected to be negligible. It may become the limiting factor if the $\nu_{\mu}-\nu_{\tau}$ oscillation experiment is ever pushed to even lower values of $\sin ^{2} 2 \theta_{\tau \mu}$. The background due to "direct $\nu_{\tau}$ " can, in principle, be measured by turning down, removing or diverting the focused neutrino beam. At lower energies (such as at CERN), the $F$ background is smaller than at higher energies (such as at Fermilab).

We conclude that the proposed experiment is difficult, but not impossible. The potential reward is, in our opinion, extremely significant.

If the experiment is performed and oscillations are found, it will provide us with information on $m\left(\nu_{\tau}\right)$. A precise determination of $m\left(\nu_{\tau}\right)$ may require additional, more complicated, experiments at different distances and/or energies. However, the existence of any $\nu_{\mu}-\nu_{\tau}$ oscillations in an experiment of the type discussed here, would indicate that $m\left(\nu_{\tau}\right)$ is at least a few $e V^{\prime} s$, making it a very likely candidate for the dark matter. If $m\left(\nu_{\tau}\right)$ is found to be in the appropriate mass range, it is probably the cosmological dark matter of the universe and it becomes the dominant contributor to its energy! This would correspond to our solution II in section 6 .

If the result is negative down to $\sin ^{2} 2 \theta_{\tau \mu} \approx 4 \cdot 10^{-4}$ and if, like E531, the experiment is sensitive to $m\left(\nu_{\tau}\right)$-values as low as a few $\mathrm{eV}$, we face two possibilities: The most likely one is that $m\left(\nu_{\tau}\right)$ is at, or below, few $e V$ and it does not form the cosmological dark matter of the universe. In that case, $m\left(\nu_{\mu}\right)$ is most likely to be at, or below, $10^{-2} \mathrm{eV}$, just the range required for explaining the solar neutrino puzzle by $\nu_{\mu}-\nu_{e}$ oscillations $^{[15-16]}$. This would correspond to solution III, and, of course, solution IV may also be possible.

The second possibility (in the case of a negative result) is that $\nu_{\tau}$ is still around $15-65 \mathrm{eV}$, but for some peculiar reason $\theta_{\tau \mu}<0.01$, well below the analogous quark angles and possibly even below the angle $\theta_{13}^{(q)}$. This would be a very small angle and it is not suggested by any known model. However, such a situation cannot be ruled out and the only way to cope with it would be to push the experiment even further, to lower values of $\sin ^{2} 2 \theta_{\tau \mu}$.

If $m\left(\nu_{\tau}\right)$ is in the $15-65 \mathrm{eV}$ range, $m\left(\nu_{\mu}\right)$ is likely to be approximately around $0.1 \mathrm{eV}$. In such a case, $\nu_{\mu}-\nu_{e}$ oscillations at $\Delta m^{2} \approx 10^{-2} \mathrm{eV}$ become relevant. Such experiments are being now contemplated. However, even if $\nu_{\mu}-\nu_{e}$ oscillations are discovered at $m\left(\nu_{\mu}\right) \approx 0.1 \mathrm{eV}$, we still cannot be sure that $\nu_{\tau}$ is the cosmological dark matter. Only a direct observation of $\nu_{\tau}-\nu_{\mu}$ oscillations 
will be convincing.

We summarize: the experiment described in detail in this section is designed to test solution II (as presented in section 6). We believe that it is a crucial experiment. We hope it is done in the near future.

\section{References}

1. Harari, H. and Nir, Y., Nucl. Phys. B292, 251 (1987).

2. Harari, H., Proceedings of the "Neutrino 88" Conference, Medford, Mass., U.S.A., June, 1988.

3. Harari, H., Phys. Lett., 216B, 413 (1989).

4. Yanagida, T., in Proc. Workshop on Unified Theory and Baryon Number in the Universe, eds. O. Sawada and A. Sugamoto, (KEK, 1979):

Gell-Mann, M., Ramond, P. and Slansky, R, in Supergravity, eds. P. van Nieuwenhuizen and D. Freedman (North-Holland, 1980).

5. Fritsch, M. et al., Phys. Lett. 173B, 485 (1986); Abela, R. et al., Phys. Lett. 146B, 431 (1984); Albrecht, H. et al., Phys. Lett. 202B, 149 (1988).

6. Steigman, G., Schramm, D.N. and Gunn, J.E., Phys. Lett. ㅌ66, 202 (1973).

7. Glashow, S.L., Proc. Rencontres de Physique de la Vallee d'Aosta, La Thuile, Italy, 1987. See also reference 1.

8. Wolfenstein, L., Phys. Rev. D17, 2369 (1978); Mikheyev, S.P. and Smirnov, A.Yu., in Proc. 10th Int. Workshop on Weak Interactions, Savonlinna, Finland (1985).

9. Davis, R., Proceedings of the "Neutrino 88" Conference.

10. Bethe, H.A. Phys. Rev. Lett. 56, 1305 (1986).

11. Rosen, S.P. and Gelb, J.M., Phys. Rev. D34, 969 (1986).

12. For various reviews of dark matter see e.g. Proc. of the 1986 Jerusalem Winter School on Dark Matter, eds. J.N. Bahcall, T. Piran and S. Wein berg.

13. Gerstein, S.S. and Zeldovich, Ya.B., JETP Lett. $\underline{4}, 120$ (1966); Cowsik, R. and McClelland, J., Phys. Rev. Lett. 29, 669 (1972).

14. Lee, B.W. and Weinberg, S., Phys. Rev. Lett. $\underline{39}, 165$ (1977); Hut, P. Phys. Lett. 69B, 85 (1977).

15. Dicus, D.A., Kolb, E.W., and Teplitz, V.L., Phys. Rev. Lett. $\underline{39}, 168$ (1977); (E)39, 973 (1977).

16. Pal, P.B. and Wolfenstein, L., Phys. Rev. D23, 766 (1982); Nieves, J.F., Phys. Rev. D28, 1664 (1983).

17. Hosotani, Y., Nucl. Phys. 191, 411 (1981).

18. For a recent review of neutrino oscillations see e.g. R.A. Eichler, Proc. of the International Symposium on Lepton Photon Interactions at High Energies, Hamburg, 1987. 
19. E531 Collaboratio, N. Ushida et al., Phys. Rev. Lett. $\underline{57}, 289$ (1986).

20. See V. Zacek, Proceedings of the Rencontre de Moriond, January 1988, to be published. 